\title{
An Automatic Detection Model of Microaneurysms Based on Improved FC-Densenet
}

\author{
Zhenhua Wang \\ Shanghai Ocean University \\ Mudi Yao \\ Nanjing Medical University \\ Xiaokai Li \\ Shanghai Ocean University \\ Qing Jiang \\ Nanjing Medical University \\ Biao Yan ( $\sim$ yanbiao1982@hotmail.com) \\ Fudan University
}

\section{Research Article}

Keywords: Micro aneurysms, Fluorescein fundus angiography images, FC-DenseNet, Detection model

Posted Date: July 9th, 2021

DOl: https://doi.org/10.21203/rs.3.rs-677644/v1

License: (c) (1) This work is licensed under a Creative Commons Attribution 4.0 International License. Read Full License 
1 An automatic detection model of microaneurysms based on improved FC-DenseNet

${ }^{1}$ College of Information Science, Shanghai Ocean University, Shanghai 201306, China

${ }^{2}$ The Affiliated Eye Hospital, Nanjing Medical University, Nanjing, 211166, China

${ }^{3}$ Eye Institute, Eye \& ENT Hospital, Shanghai Medical College, Fudan University, Shanghai 200030, China

${ }^{*}$ These authors contributed equally to this work.

\section{Correspondence to:}

\section{Biao Yan}

21 Fudan University $83^{\#}$ Fen Yang Road, Shanghai, China 200030

22 E-mail: yanbiao1982@ hotmail.com

Tel: 86-21-64377134 Fax: 86-21-64377134 


\section{ABSTRACT}

Diabetic retinopathy (DR) is a common eye disease, which leads cause of blindness all around the world.

41 Microaneurysms (MAs) is one of the early symptoms of DR. Accurate and effective MAs detection and 42 segmentation is an important step for the diagnosis and treatment of DR. In this paper, we propose an automatic model for detection of MAs in fluorescein fundus angiography (FFA) images. The model mainly consists of two steps. The first step is pre-processing of FFA images, where the quality of FFA images is improved by Histogram Stretching and Gaussian Filtering algorithm. The second step is to detect MAs regions, where the MAs regions are detected by improved FC-DenseNet. We compare the proposed model with traditional FCDenseNet model and other previously published models. The experimental result shows that our proposed model has the highest scores on evaluation metrics of pixel accuracy (PA), mean pixel accuracy (MPA), precision (Pre), recall $(R e)$, F1-score $(F 1)$ and mean intersection over union $(M I o U)$, which are 99.97\%, 94.19\%, 88.40\%, $89.70 \%, 88.98 \%$ and $90.14 \%$, respectively. The result suggests that the performance of our proposed model is closer to the ground truth of MAs detection. Our proposed model would be helpful for ophthalmologists to find the symptoms more quickly and to take better treatment measures in the screening process of diabetic retinopathy.

Key Words: Micro aneurysms; Fluorescein fundus angiography images; FC-DenseNet; Detection model

\section{Introduction}

Retinal microaneurysms (MAs) is defined as small swelling of tiny blood vessels, which mainly locates in the inner nuclear layer and deep capillary layer. It often occurs as early clinical signs of diverse retinal or systemic diseases, including diabetic retinopathy (DR), retinal vein occlusions, infectious and so on. The number and turnover of retinal MAs are considered as indicators to assess the presence, severity, and progression risk of related retinopathy. Thus, early handling of MAs is needed to prevent vision loss caused by these retinopathies, especially DR. MAs can be identified by many modern imaging technologies including color fundus photography, fundus fluorescein angiography (FFA) and optical coherence tomography angiography (OCTA). Clinically, FFA is well-recognized as the gold standard to visualize retinal vasculature and routinely used to describe the subtle vascular alterations.

FFA is highly sensitive and demonstrates MAs as a hyperfluorescent dots in the early phase, contributing to 66 identification or evaluation of related retinal diseases. It is an important imaging modality, which captured after 67 intravenous injection of fluorescein dye using the dedicated fundus camera equipped with excitation and barrier 
filters. However, there are still some limitations in clinical applications. First, manual detection and quantification of MAs are labor-intensive and time-consuming. With the increasing amount of FFA images that require analysis, there is no sufficient number of ophthalmologists, especially in some rural areas. Second, such manual work is subjective and error-prone, leading to poor reproducibility. Moreover, it is also infeasible for large-scale FFA 72 image analysis.

73 Therefore, the automated computer system may be helpful for ophthalmologists to identify and assess MAs more efficiently. Numerous methodologies have been proposed currently. Zhang et al. [1] presented a novel MA detection method based on a deep neural network with a multilayer attention mechanism for retinal fundus images. Mazlan et al. [2] proposed an automatic detection of microaneurysms (MAs) in the fundus retina images. Firstly, the images were filtered and the contrast enhanced. Then, the images were segmented using H-maxima and thresholding technique. Long et al. [3] proposed a microaneurysms' detection method using machine learning based on directional local contrast (DLC) for the early diagnosis of DR. Sarhan et al. [4] proposed a two-stage deep learning approach for microaneurysms segmentation using multiple scales of the input with selective sampling and embedding triplet loss. Yang et al. [5] proposed a method based on improved Hessian matrix eigenvalue analysis to detect microaneurysms and hemorrhages in the fundus images of diabetic patients. Kou et al. [6] proposed an architecture for U-Net, named deep recurrent U-Net (DRU-Net), obtained by combining the deep residual model and recurrent convolutional operations into U-Net.

Various factors can affect the performance of automatic MAs detection model, especially the low contrast, the tiny size and the similarity with blood vessels of MAs. Most presented studies on MAs detection were based on traditional detection models. However, the traditional detection models usually have low efficiency for MAs detection, owing to MAs are relatively small circular structure in FFA image. Some models based on neural network did not achieve better detection for MAs, such as GoogleNet[7] and ResNet[8], only learn higher-level features from the features of the upper layer forward, and then give up the features of the lower layer after abstracting them. These neural networks do not make full use of the characteristic information of the context, which led to the problem of false detection for small target. Thus, the main purpose of our work is to develop an efficient and accurate automatic MAs detection model in retinal FFA images, which has improved the imbalance 

FC-DenseNet.

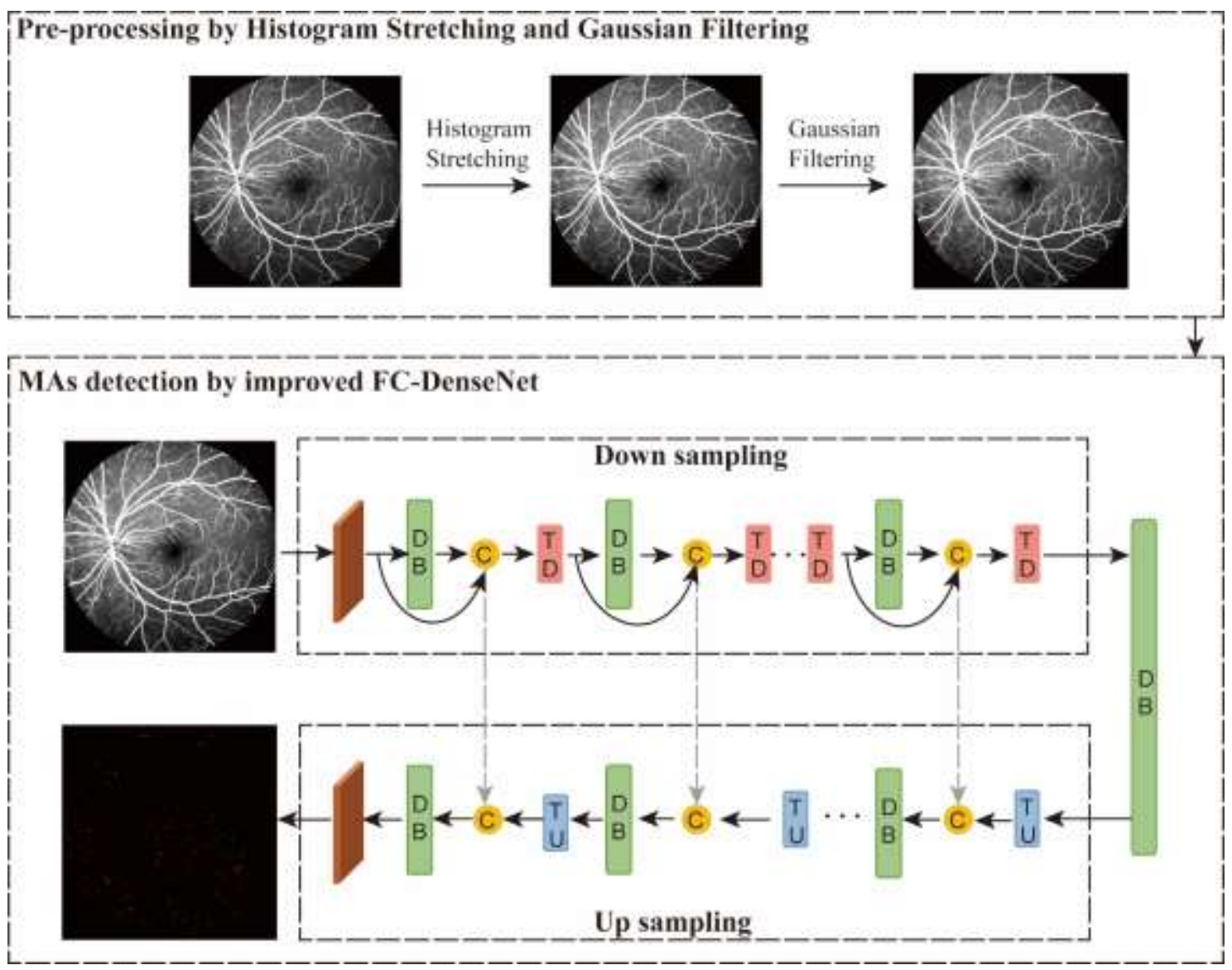

Figure. 1. Flowchart of MAs-FC-DenseNet model

\section{Pre-processing by Histogram Stretching and Gaussian Filtering}

Due to the low contrast and high noise of FFA images, it is difficult to identify the MAs features of FFA images. In the pre-processing step, the quality of FFA images is improved. Firstly, the contrast between MAs and the background is enhanced by Histogram Stretching algorithm[9]. Then the noises of the FFA images are reduced by Gaussian Filtering[10].

Histogram Stretching is expressed as

$I_{\text {new }}=\left(\frac{G_{\text {max }}-G_{\text {min }}}{I_{\text {max }}-I_{\text {min }}}\right)\left(I-I_{\text {min }}\right)+G_{\text {min }}$

Where $I_{\text {new }}$ is the new transformed image, $I_{\max }$ and $I_{\min }$ are the largest and smallest possible grey level value in the original image, respectively. $G_{\max }$ and $G_{\min }$ are the largest and smallest possible grey level value in the transformed image respectively. 
Where $\sigma^{2}$ is the variance of Gaussian Filtering, $l$ is the size of the filter kernel.

\section{MAs detection by improved FC-DenseNet}

120 At this step, retinal microaneurysms (MAs) are detected by improved Fully Convolutional DenseNet (FC-DenseNet)

121 [11]. FC-DenseNet contains a down sampling path for extracting sparse semantic features and an up sampling path for 122 restoring original resolution. Down sampling path consists of dense block(DB) layer [12] and transition down(TD) layer. 123 Moreover, up sampling path consists of DB layer and transition up(TU) layer. DB layer is composed of batch normalization(BN)[13], ReLU[14], $3 \times 3$ convolution and dropout with probility $p=0.2$.TD layer is composed of $\mathrm{BN}$,

125 ReLU, $1 \times 1$ convolution, dropout with probility $p=0.2$ and $2 \times 2$ maximum pooling. TU layer includes $3 \times 3$ transposed 126 convolution with stride 2.

127 As shown in Figure 1, the feature maps from the down sampling path are concatenated with the corresponding feature maps in the up sampling path. Note that the connectivity pattern in the up sampling and the down sampling paths are different. In the down sampling path, the input to a dense block is concatenated with its output, leading to a linear growth of the number of feature maps, whereas in the up sampling path, it is not.

131 Due to the feature of MAs, small and less in FFA images, it is imbalance between MAs and background and difficult

132 to detect accurately. Here, we replace the cross entropy loss of FC-DenseNet with the focal loss[15]. The focal loss can 133 decrease the weight of the background and increase the weight of MAs, so that the model can focus more on the MAs 134 regions in training and improve the accuracy of MAs detection.

135 Here, the focal loss function is expressed as

$F L\left(p_{t}\right)=-\alpha_{t}\left(1-p_{t}\right)^{\gamma} \log \left(p_{t}\right)$

137 Where $p_{t}$ is probability of correct prediction for different categories. $\alpha_{t}$ and $\gamma \geq 0$ are adjustable hyperparameters, 138 both of them are used to control the sharing weight of different samples to the total loss.

\subsection{Materials}

\section{Datasets}

142 In this study, we construct the large FFA images cohort with the collaboration of Nanjing Eye Hospital, Suzhou First 
144 from 65 eyes (34 left eyes and 31 right eyes) of 60 patients of DR (age range:31-81 years old; male and female ratio1:5)

145 who underwent FFA in the Affiliated Eye Hospital of Nanjing Medical University from August 2015 to December 2019.

146 The operations are performed by 3 experienced ophthalmologists using Heidelberg Retina Angiograph (Heidelberg

147 Engineering, Heidelberg, Germany) with a $30^{\circ}$ field of view. This study is approved by Ethics Committee of the

148 author's institute and followed the tenets of the Declaration of Helsinki and. Written consent is given by the patients for

149 the information to be stored in the hospital database. Subsets of 960, 120 and 120 FFA images are randomly selected for

150 training, validation and testing, respectively. Three retinal ophthalmologists with more than 10-year clinical experience

151 work individually to label MAs images as reference. A senior expert is consulted in case of disagreement.

\section{Evaluation metrics}

153 Six different metrics, including pixel accuracy $(P A)[16]$, mean pixel accuracy $(M P A)[16]$, Precision (Pre)[17], Recall

$154(R e)[17]$, F1-score $(F 1)[18]$ and mean intersection over union $(M I o U)[19]$ are calculated to estimate the performance of

155 MAs detection.

156

$P A=\frac{\sum_{i=0}^{k} p_{i i}}{\sum_{i=0}^{k} \sum_{j=0}^{k} p_{i j}}$

$157 \quad M P A=\frac{1}{k+1} \sum_{i=0}^{k} \frac{p_{i i}}{\sum_{j=0}^{k} p_{i j}}$

$158 \quad$ Pre $=\frac{T P}{T P+F P}$

$159 R e=\frac{T P}{T P+F N}$

$160 \quad F 1=\frac{2 \times \operatorname{Pre} \times \operatorname{Re}}{\operatorname{Pre}+\operatorname{Re}}$

$M I o U=\frac{1}{k+1} \sum_{i=0}^{k} \frac{p_{i i}}{\sum_{j=0}^{k} p_{i j}+\sum_{j=0}^{k} p_{j i}-p_{i i}}$

Where $T P, F P$ and $F N$ denote the true positive region, false positive region and false negative region, respectively. $k=1$ is the number of classes in the ground truth, $p_{i j}$ is the number of pixels of class $i$ predicted as class $j$. The $P A$

164 calculates the overall pixel accuracy. The MPA measures the average pixel accuracy of MAs and background. The Pre 165 and $R e$ measure the proportion of real MAs in samples predicted as MAs and the proportion of correct predictions in all MAs, respectively. F1-score (F1) is a balanced metric and determined by precision and sensitivity simultaneously. Mean 
intersection over union $(M I o U)$ is an accuracy assessment metric applied to measure the similarity between ground truth

168

169

170

and prediction.

\section{Implementation}

The following hardware configuration is required for all experiments to be carried out: Ubuntu 16.04.4, 2GPUs, GPU model is NVIDIA Tesla P100 PCIE, 1 GPU memory is 16G. Software environment is required for the experiment: deep-learning framework Tensorflow1.8.0, programming experiment with python3.6.

\section{Experimental Results}

The original FFA images and the detection results by our proposed model (MAs-FC-DenseNet) and the ground truth by the retinal ophthalmologists are shown in Figure 2. To evaluate the performance on the detection of retinal microaneurysms, two comparison experiments are conducted. In experiment 1, our proposed model, MAs-FC-DenseNet, is compared against the traditional FC-DenseNet models including FC-DenseNet56[11] and FC-DenseNet67[11]. In experiment 2, our proposed model is compared against other end-to-end models, including DeeplabV3+[20] and PSPNet[21] models.
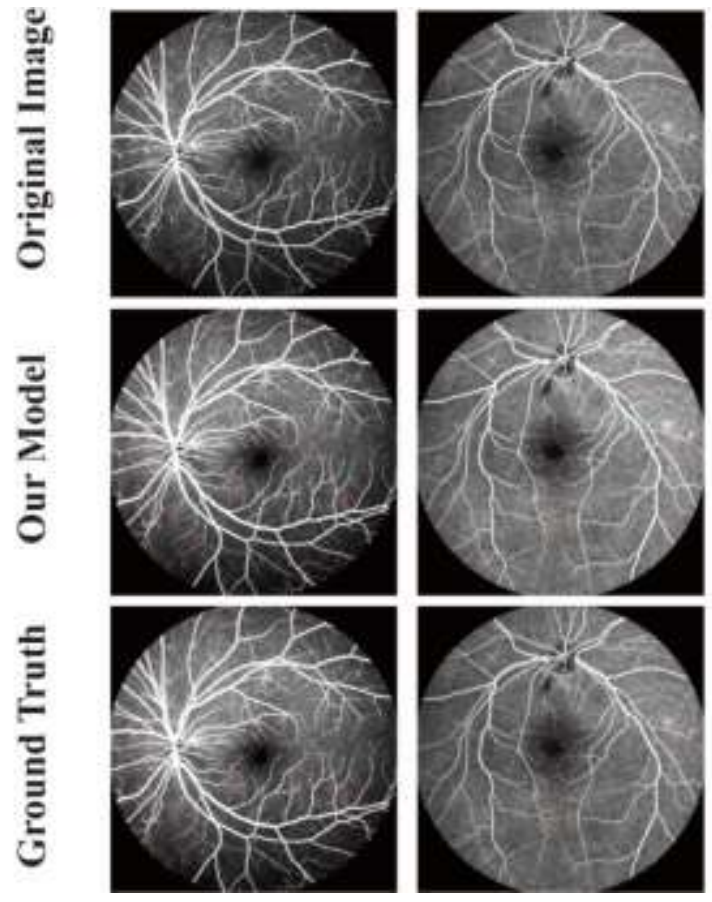
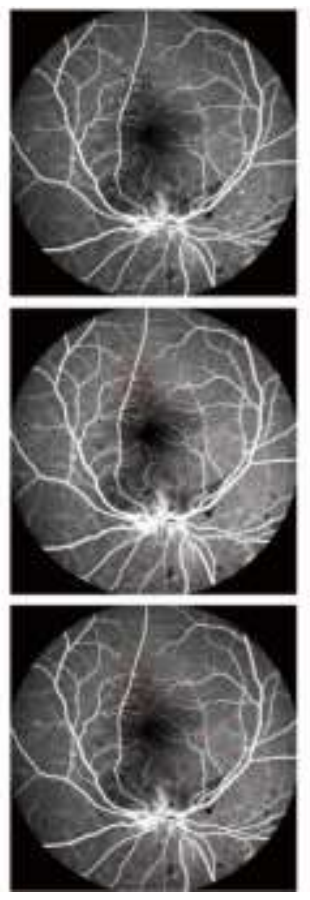
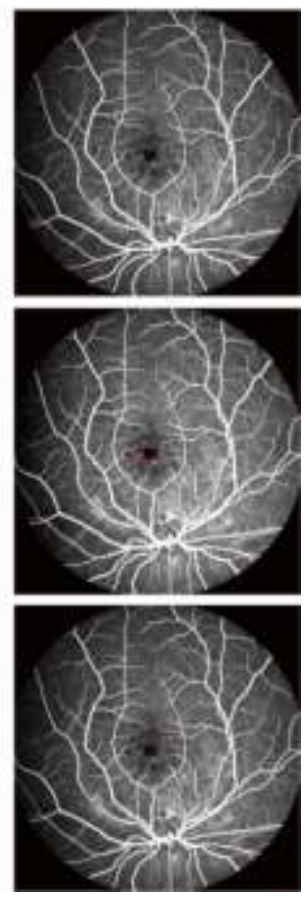

Figure 2. Original FFA image, and the detection results of MAs by the proposed models and the ground truth.

\section{1 Compared with traditional FC-DenseNet models}

We compared our proposed model against the traditional FC-DenseNet models, including FC-DenseNet 56 and FC-DenseNet 67, to evaluate the detection performance of MAs. The detection results of MAs are shown in Figure 3.and the results of evaluation metrics is shown in Table 1. 

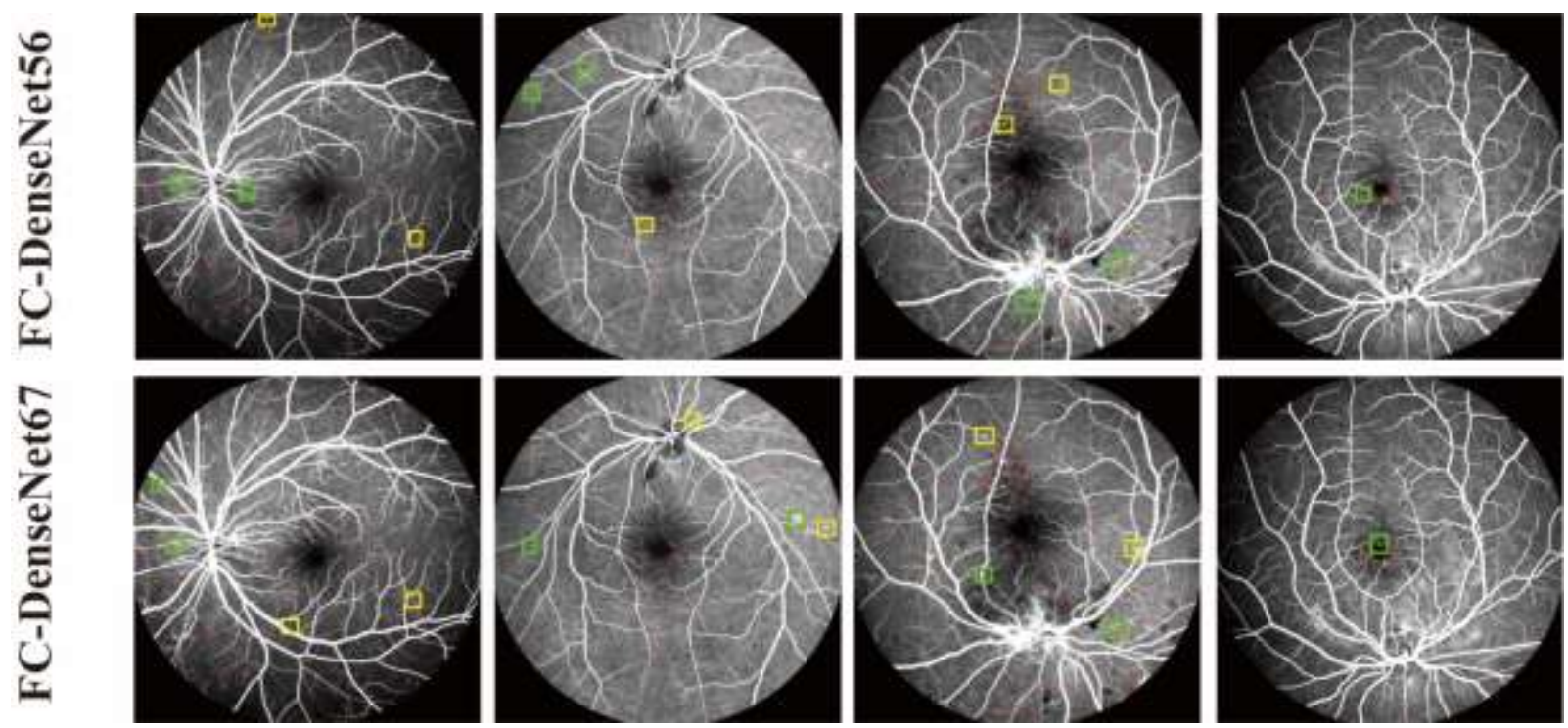

Figure 3. Detection results of MAs by traditional FC-DenseNet models.

Table 1. Performance comparison between traditional FC-DenseNet models and our model.

\begin{tabular}{cllllll}
\hline \multirow{2}{*}{ Model } & \multicolumn{5}{c}{ Evaluation metrics } \\
\cline { 2 - 7 } & $\boldsymbol{P A ( \% )}$ & $\boldsymbol{M P A}(\%)$ & $\boldsymbol{P r e}(\%)$ & $\boldsymbol{R e}(\%)$ & $\boldsymbol{F 1}(\%)$ & $\boldsymbol{M I o U}(\%)$ \\
\hline \multirow{2}{*}{ FC-DenseNet56 } & $99.96 \pm 0.02$ & $88.74 \pm 0.11$ & $77.49 \pm 0.17$ & $80.87 \pm 0.10$ & $78.07 \pm 0.16$ & $82.30 \pm 0.09$ \\
FC-DenseNet67 & $99.96 \pm 0.02$ & $91.37 \pm 0.06$ & $82.73 \pm 0.09$ & $80.94 \pm 0.11$ & $81.12 \pm 0.10$ & $84.34 \pm 0.09$ \\
Our Model & $\mathbf{9 9 . 9 7} \pm \mathbf{0 . 0 1}$ & $\mathbf{9 4 . 1 9} \pm \mathbf{0 . 0 4}$ & $\mathbf{8 8 . 4 0} \pm \mathbf{0 . 0 6}$ & $\mathbf{8 9 . 7 0} \pm \mathbf{0 . 0 5}$ & $\mathbf{8 8 . 9 8} \pm \mathbf{0 . 0 6}$ & $\mathbf{9 0 . 1 4} \pm \mathbf{0 . 0 5}$ \\
\hline
\end{tabular}

Based on Figure 3 and Table 1, we can find that there are more missed detection and false detection in the detection results by FC-DenseNet56 and FC-DenseNet67 model, as the green mark and yellow mark shown in Figure 3. Compared with the traditional FC-DenseNet models, the performance of our proposed model, MAs-FC-DenseNet, is closer to the ground truth. In addition, our proposed model achieves higher scores of PA, MPA, Pre, Re, F1 and MIoU, which are $99.97 \%(0.01 \uparrow), \quad 94.19 \%(2.82 \uparrow), 88.40 \%(5.67 \uparrow), 89.70 \%(8.76 \uparrow), \quad 88.98 \%(7.86 \uparrow)$ and $90.14 \%(5.80 \uparrow)$, respectively.

\section{2 Compared with other end-to-end models}

We compared our proposed model against other end-to-end models, including deeplabV3+ and PSPNet, to evaluate the detection performance of MAs. The detection results of MAs are shown in Figure 4.and the results of evaluation metrics is shown in Table 2. 

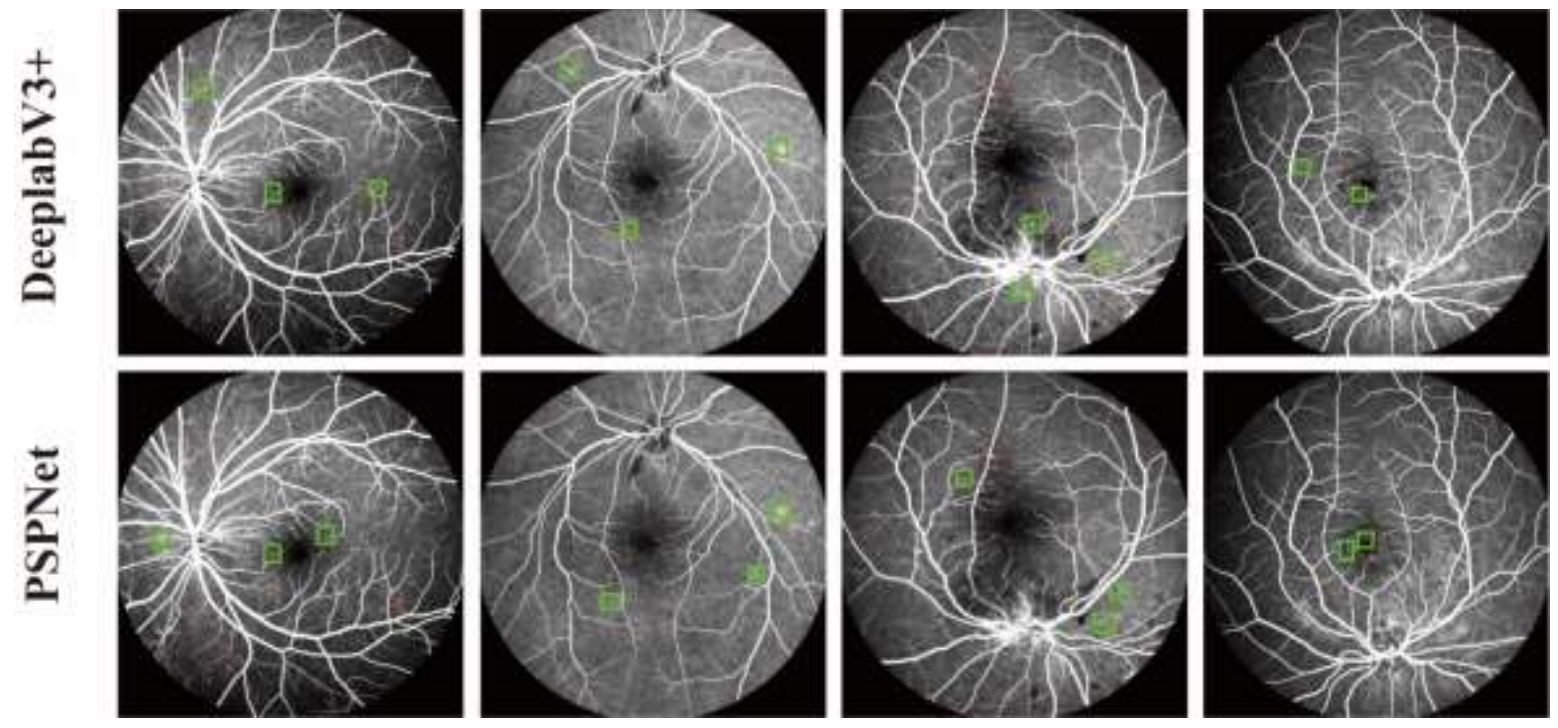

205

206

207

208

209

210

Figure 4. Detection results of MAs by end-to-end models.

Table 2. Performance comparison between different end-to-end models.

\begin{tabular}{cllllll}
\hline \multirow{2}{*}{ Model } & \multicolumn{5}{c}{ Evaluation metrics } \\
\cline { 2 - 7 } & $\boldsymbol{P A ( \% )}$ & $\boldsymbol{M P A}(\boldsymbol{\%})$ & $\boldsymbol{P r e}(\%)$ & $\boldsymbol{R e}(\boldsymbol{\%})$ & $\boldsymbol{F 1}(\%)$ & $\boldsymbol{M I o U}(\boldsymbol{\%})$ \\
\hline DeeplabV3+ & $99.91 \pm 0.04$ & $71.03 \pm 0.19$ & $42.08 \pm 0.27$ & $69.36 \pm 0.19$ & $51.12 \pm 0.26$ & $67.60 \pm 0.21$ \\
PSPNet & $99.93 \pm 0.03$ & $81.85 \pm 0.14$ & $63.73 \pm 0.21$ & $76.00 \pm 0.12$ & $66.96 \pm 0.20$ & $75.46 \pm 0.18$ \\
Our Model & $\mathbf{9 9 . 9 7} \pm \mathbf{0 . 0 1}$ & $\mathbf{9 4 . 1 9} \pm \mathbf{0 . 0 4}$ & $\mathbf{8 8 . 4 0} \pm \mathbf{0 . 0 6}$ & $\mathbf{8 9 . 7 0} \pm \mathbf{0 . 0 5}$ & $\mathbf{8 8 . 9 8} \pm \mathbf{0 . 0 6}$ & $\mathbf{9 0 . 1 4} \pm \mathbf{0 . 0 5}$ \\
\hline
\end{tabular}

Based on Figure 4 and Table 2, we can find that the DeeplabV3+ model could not detect the boundaries of MAs region and normal region well, and there are some false detection, as the green mark shown in Figure 4. At the same time, PSPNet model has inferior accuracy of detection result, and omitted some MAs regions, as the green mark shown in Figure 4. In contrast, the detection results of our proposed model shows great consistency with the ground truth. Moreover, our proposed model, MAs-FC-DenseNet, achieves the highest scores of PA, MPA, Pre, Re, F1 and MIoU than other models.

\section{Discussion}

Microaneurysms are the first clinically observable manifestations of diabetic retinopathy. Early diagnosis and timely intervention can halt or reverse the progression of this disease[22]. In this paper, we propose a two-step model, MAs-FC-DenseNet, for automatic detection of MAs in FFA images. Firstly, the pre-processing of FFA images is conducted to enhance the contrast and to reduce the noise of FFA images. Then, MAs are detected by our improved FC-DenseNet. 
Most FFA images suffer from high noise and low contrast. Moreover, in FFA images, it is difficult to distinguish MAs from blood vessels. Therefore, we improve the quality of FFA images by Histogram Stretching and Gaussian Filtering in the pre-processing step. Due to the features of MAs, small and less in FFA images, it is difficult to detect MAs accurately. Therefore, we use the FC-DenseNet model to detect the deep features of MAs, and use the focal loss to enhance the detection accuracy of MAs in the detection step.

Compared with other deep learning network models, such as DeeplabV3+ and PSPNet, it could be found that our proposed model achieves higher precision. The evaluation metrics, pixel accuracy $(P A)$, mean pixel accuracy $(M P A)$, Precision (Pre), Recall (Re), F1-score (F1), and mean intersection over union (MIoU), get the highest score, which are $99.97 \%, 94.19 \%, 88.40 \%, 89.70 \%, 88.98 \%$ and $90.14 \%$, respectively. The detection results of our model is closer to the detection results by retinal ophthalmologists.

Our proposed model, MAs-FC-DenseNet, can efficiently and accurately detect MAs in FFA images. However, there are still some shortcomings in the clinical applications. For instance, how to calculate the area of MAs and how to count the number of MAs are also challenges, which are important information for diagnosis and treatment 236 of diseases.

To summarize, this paper proposes a two-step model, MAs-FC-DenseNet, for automatic detection of MAs in FFA images. Firstly, the pre-progressing of FFA images is conducted, where the quality of FFA images are enhanced by Histogram Stretching and Gaussian Filtering. Then, the MAs are detected by the improved FCDenseNet. This automatic model can supply importance information for ophthalmologists to detect MAs disease and enhance the efficiency of several ocular diseases, especially DR.

\section{Data Availability}

244 The data used to support the findings of this study are included within the article.

\section{Conflicts of Interest}

The author(s) declare(s) that there is no conflict of interest regarding the publication of this paper.

\section{Authors' Contributions}

Biao Yan and Qing Jiang were responsible for the conceptualization, data collection. Zhen-Hua Wang and Xiao-Kai Li were responsible for the experiment design and manuscript writing; Mu-Di Yao conducted the data collection and data entry; Biao Yan and Zhen-Hua Wang were responsible for overall supervision and manuscript revision 


\section{Acknowledgments}

This research was generously supported by the grants from the National Natural Science Foundation of China (Grant No. 814705942).

\section{References}

[1] L. Z. Zhang, S. X. Feng, G. D. Duan, Y. Li, and G. S. Liu, "Detection of Microaneurysms in Fundus Images Based on an Attention Mechanism," (in English), Genes, Article vol. 10, no. 10, p. 19, Oct 2019, Art. no. 817.

[2] N. Mazlan, H. Yazid, H. Arof, and H. M. Isa, "Automated Microaneurysms Detection and Classification using Multilevel Thresholding and Multilayer Perceptron," (in English), Journal of Medical and Biological Engineering, Article vol. 40, no. 2, pp. 292-306, Apr 2020.

[3] S. C. Long, J. L. Chen, A. T. Hu, H. P. Liu, Z. Q. Chen, and D. C. Zheng, "Microaneurysms detection in color fundus images using machine learning based on directional local contrast," (in English), Biomedical Engineering Online, Article vol. 19, no. 1, p. 23, Apr 2020, Art. no. 21.

[4] M. H. Sarhan, S. Albarqouni, M. Yigitsoy, N. Navab, and E. Abouzar, "Microaneurysms segmentation and diabetic retinopathy detection by learning discriminative representations," (in English), Iet Image Processing, Article vol. 14, no. 17, pp. 4571-4578, Dec 2020.

[5] L. Y. Yang, S. J. Yan, and Y. Z. Xie, "Detection of microaneurysms and hemorrhages based on improved Hessian matrix," (in English), International Journal of Computer Assisted Radiology and Surgery, Article vol. 16, no. 6, pp. 883-894, Jun 2021.

[6] C. Kou, W. Li, W. Liang, Z. Yu, and J. Hao, "Microaneurysms segmentation with a U-Net based on recurrent residual convolutional neural network," (in English), Journal of medical imaging (Bellingham, Wash.), vol. 6, no. 2, p. 025008, 2019 Apr (Epub 2019 Jun 2019.

[7] J. L. Yu, A. W. Schumann, S. M. Sharpe, X. H. Li, and N. S. Boyd, "Detection of grassy weeds in bermudagrass with deep convolutional neural networks," (in English), Weed Science, Article vol. 68, no. 5, pp. 545-552, Sep 2020, Art. no. Pii s0043174520000466.

[8] Y. Lu, X. J. Qin, H. Y. Fan, T. T. Lai, and Z. Y. Li, "WBC-Net: A white blood cell segmentation network based on UNet plus plus and ResNet," (in English), Applied Soft Computing, Article vol. 101, p. 11, Mar 2021, Art. no. 107006.

[9] W. L. Luo, S. Q. Duan, and J. W. Zheng, "Underwater Image Restoration and Enhancement Based on a Fusion Algorithm With Color Balance, Contrast Optimization, and Histogram Stretching," (in English), Ieee Access, Article vol. 9, pp. 31792-31804, 2021.

[10] M. Nasor and W. Obaid, "Segmentation of osteosarcoma in MRI images by K-means clustering, Chan-Vese segmentation, and iterative Gaussian filtering," (in English), Iet Image Processing, Article vol. 15, no. 6, pp. 1310-1318, May 2021.

[11] X. J. Guo, Z. H. Chen, and C. Y. Wang, "Fully convolutional DenseNet with adversarial training for semantic segmentation of high-resolution remote sensing images," (in English), Journal of Applied Remote Sensing, Article vol. 15, no. 1, p. 12, Mar 2021, Art. no. 016520.

[12] S. Brahimi, N. Ben Aoun, A. Benoit, P. Lambert, and C. Ben Amar, "Semantic segmentation using reinforced fully convolutional densenet with multiscale kernel," (in English), Multimedia Tools and Applications, Article vol. 78, no. 15, pp. 22077-22098, Aug 2019.

[13] S. Lee and C. Lee, "Revisiting spatial dropout for regularizing convolutional neural networks," (in English), Multimedia Tools and Applications, Article vol. 79, no. 45-46, pp. 34195-34207, Dec 2020.

[14] A. Soltani and S. Nasri, "Improved algorithm for multiple sclerosis diagnosis in MRI using convolutional neural network," (in English), Iet Image Processing, Article vol. 14, no. 17, pp. 45074512, Dec 2020.

[15] T. F. Romdhane, H. Alhichri, R. Ouni, and M. Atri, "Electrocardiogram heartbeat classification based on a deep convolutional neural network and focal loss," (in English), Computers in Biology and Medicine, Article vol. 123, p. 13, Aug 2020, Art. no. 103866.

[16] H. B. Deng, T. Y. Xu, Y. C. Zhou, and T. Miao, "Depth Density Achieves a Better Result for Semantic Segmentation with the Kinect System," (in English), Sensors, Article vol. 20, no. 3, p. 14, Feb 2020, Art. no. 812. 
[17] X. L. Zhu, Z. Y. Cheng, S. Wang, X. J. Chen, and G. Q. Lu, "Coronary angiography image segmentation based on PSPNet," (in English), Computer Methods and Programs in Biomedicine, Article vol. 200, p. 8, Mar 2021, Art. no. 105897.

[18] D. Chicco and G. J. B. g. Jurman, "The advantages of the Matthews correlation coefficient (MCC) over F1 score and accuracy in binary classification evaluation," vol. 21, no. 1, pp. 1-13, 2020.

[19] Z. Li and Y. Xia, "Deep Reinforcement Learning for Weakly-Supervised Lymph Node Segmentation in CT Images," (in English), Ieee Journal of Biomedical and Health Informatics, Article vol. 25, no. 3, pp. 774-783, Mar 2021.

[20] M. Liu et al., "Comparison of multi-source satellite images for classifying marsh vegetation using DeepLabV3 Plus deep learning algorithm," (in English), Ecological Indicators, Article vol. 125, p. 21, Jun 2021, Art. no. 107562.

[21] A. M. Shaaban, N. M. Salem, and W. I. Al-atabany, "A Semantic-based Scene segmentation using convolutional neural networks," (in English), Aeu-International Journal of Electronics and Communications, Article; Proceedings Paper vol. 125, p. 9, Oct 2020, Art. no. 153364.

[22] A. Bilal, G. Sun, and S. Mazhar, "Survey on recent developments in automatic detection of diabetic retinopathy," (in English), Journal Francais D Ophtalmologie, Review vol. 44, no. 3, pp. 420-440, Mar 2021.

\section{Acknowledgements}

This work was generously supported by the grants from the National Natural Science Foundation of China (Grant No. 814705942).

\section{Author contributions statement}

B.Y. was responsible for the conceptualization, data collection. Z.W. was responsible for supervision and manuscript writing. M.Y. conducted the data collection and data entry; Z.W. and X.L. were responsible for the experiment design. All authors read and approved the final manuscript.

\section{Competing interests}

The author(s) declare(s) that there is no conflict of interest regarding the publication of this paper. 